\title{
Factors necessary for independent walking in patients with thalamic hemorrhage
}

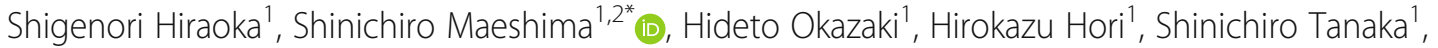
Sayaka Okamoto ${ }^{1}$, Reisuke Funahashi ${ }^{1}$, Kei Yagihashi ${ }^{1}$, Ikuko Fuse ${ }^{1}$, Naoki Asano ${ }^{1}$ and Shigeru Sonoda ${ }^{1}$

\begin{abstract}
Background: Thalamic hemorrhages cause motor paralysis, sensory impairment, and cognitive dysfunctions, all of which may significantly affect walking independence. We examined the factors related to independent walking in patients with thalamic hemorrhage who were admitted to a rehabilitation hospital.

Methods: We evaluated 128 patients with thalamic hemorrhage (75 men and 53 women; age range, 40-93 years) who were admitted to our rehabilitation hospital. The mean duration from symptom onset to rehabilitation hospital admission was $27.2 \pm 10.3$ days, and the mean rehabilitation hospital stay was $71.0 \pm 31.4$ days. Patients' neurological and cognitive functions were examined with the National Institutes of Health Stroke Scale (NIHSS) and Mini-Mental State Examination (MMSE), respectively. The relationship between patients' scores on these scales and their walking ability at discharge from the rehabilitation hospital was analyzed. Additionally, a decision-tree analysis was used to create a model for predicting independent walking upon referral to the rehabilitation hospital.

Results: Among the patients, 65 could walk independently and 63 could not. The two patient groups were significantly different in terms of age, duration from symptom onset to rehabilitation hospital admission, hematoma type, hematoma volume, neurological symptoms, and cognitive function. The decision-tree analysis revealed that the patient's age, NIHSS score, MMSE score, hematoma volume, and presence of ventricular bleeding were factors that could predict independent walking.
\end{abstract}

Conclusions: In patients with thalamic hemorrhage, the neurological symptoms, cognitive function, and neuroimaging factors at onset are useful for predicting independent walking.

Keywords: Hemorrhage, Thalamus, Outcome, Rehabilitation, Ambulation

\section{Background}

Cerebral hemorrhage occurs in $18.5 \%$ of stroke patients and thalamic hemorrhage accounts for $26 \%$ of all cerebral hemorrhages [1]. The thalamus is a vital structure that has extensive neural connections with other structures, allowing it to send signals throughout the brain including to the cerebral cortex. As such, the thalamus is involved in sensory and motor signal relays and in the regulation of consciousness. Given its interconnectedness with other regions, thalamic hemorrhages can cause cognitive dysfunctions such as aphasia, unilateral neglect, and memory impairments, as well as motor paralysis and sensory disturbances. These deficits can

\footnotetext{
* Correspondence: shinichiromaeshima@gmail.com

${ }^{1}$ Department of Rehabilitation Medicine II, School of Medicine, Fujita Health University, Tsu, Japan

${ }^{2}$ Department of Rehabilitation Medicine, Fujita Health University, Nanakuri Memorial Hospital, 114-2 Oodoricho, Tsu, Mie 514-1295, Japan
}

greatly affect a patient's ability to perform activities of daily living (ADLs) [2]. The prognosis of patients with thalamic hemorrhage varies depending on the patient's age, neurological severity, hematoma location and size, complications, and treatment type.

In rehabilitation wards, patients undergo intensive treatment in the early stages after stroke to help decrease ADL impairments and hasten recovery. Upon returning home, the reacquisition of walking ability is a major focus for patients with stroke with disabilities. However, few reports discuss the factors related to walking in patients with thalamic hemorrhage [3]. Understanding these factors is important for predicting patient outcome and for efficiently and effectively advancing their rehabilitation program.

The information available upon discharge of the patient from the acute-care hospital may be useful for 
predicting whether a patient will be able to walk independently upon discharge from the rehabilitation hospital. Here, we analyzed the factors related to independent walking in patients with thalamic hemorrhage who were admitted to a rehabilitation hospital.

\section{Methods}

\section{Patients}

From April 2013 to March 2016, 181 patients with thalamic hemorrhage visited the rehabilitation department of our hospital. After excluding patients with a history of previous stroke, neurodegenerative disease, and unconsciousness, as well as those who underwent surgical treatment or tracheotomy, we finally enrolled 128 patients $(75$ men and 53 women) in our study. The present study was conducted with the approval of the ethics committee at our university. Written informed consent was obtained from all patients or their legally acceptable representatives following a thorough explanation of the study.

\section{Evaluations}

We evaluated the following items in our patients with thalamic hemorrhage: age, duration from symptom onset to rehabilitation hospital admission, classification for hematoma location on computed tomography (CT) images [4], side of the stroke focus, hematoma volume, ventricular bleeding (yes/no), and neurological and cognitive function. The hematoma type was classified as follows: type I, hematoma localized in the thalamus; type II, hematoma extending into the internal capsule; and type III, hematoma extending into the midbrain (Fig. 1). The hematoma volume was calculated using the CT images that were acquired upon admission to the acute-care hospital as follows: major axis of the hematoma $\times$ minor axis $\times$ height $\times$ $1 / 2(\mathrm{~mL})[5]$.

Neurological severity was evaluated using the National Institutes of Health Stroke Scale (NIHSS) [6], and cognitive function was evaluated using the Mini-Mental State Examination (MMSE) [7]. At rehabilitation hospital discharge, we assessed the patients' functional ambulation category (FAC, Table 1) [8]. Here, patients were considered independent walkers if they had an FAC score $\geq 4$ (i.e., they could walk independently on level ground but required assistance with stairs and slopes). We divided the patients into two groups based on the FAC at discharge, as follows: independent-walking group $(\mathrm{FAC} \geq 4)$ and dependent-walking group (FAC $<4)$.

\section{Statistical analysis}

Data were analyzed with JMP version 12.2.0 for Macintosh (SAS Institute Inc., Cary, NC). The Mann-Whitney U test was used to test for unpaired differences between the two groups, and the independence of two factors was examined with the chi-squared test. A decision-tree analysis (Partition) was used to show the relationships among the factors that lead to independent walking and their hierarchical classification. The classification model was developed based on methods first introduced by Breiman [9]. In our model, independent walking served as the response variable and age, sex, duration from symptom onset to rehabilitation hospital admission, lesion side, hematoma volume and type, ventricular bleeding, NIHSS score, MMSE score, aphasia, and unilateral neglect served as the explanatory variables. Statistical significance was set at $\mathrm{p}<0.05$.

\section{Results \\ Demographic and clinical characteristics}

The mean age of the 128 patients with thalamic hemorrhage was $67.6 \pm 10.3$ years (range, $40-93$ years). The mean duration from symptom onset to hospitalization in the convalescent rehabilitation ward was $27.2 \pm 10.3$ days, and the mean duration of hospitalization was $71.0 \pm$ 31.4 days. The mean hematoma volume was $8.1 \pm 25.3 \mathrm{~mL}$. Regarding the disease type, CT imaging revealed that six patients were type I, 87 patients were type II, and 18 patients were type III. Ventricular bleeding was observed in 86 patients.

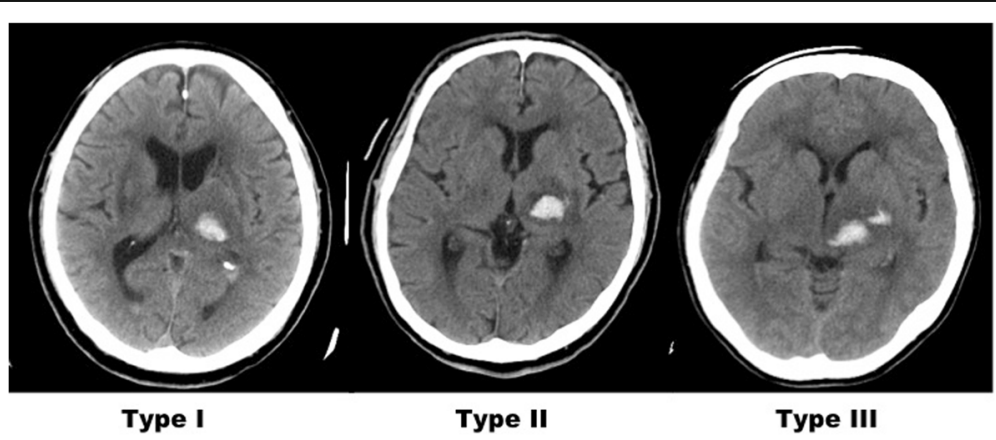

Fig. 1 Computed tomography classification of thalamic hemorrhage. The hemorrhages were classified as follows: type I, hematoma localized in the thalamus; type II, hematoma extending into the internal capsule; and type III, hematoma extending into the midbrain 
Table 1 Functional ambulation classification

Clinician-completed tick box of five broad categories of walking ability.

The categories range from independent walking outside to nonfunctional walking.

Patients are rated based on the following categories:

0 : Patient cannot walk or needs help from two or more persons.

1: Patient needs firm continuous support from one person who helps with carrying the weight and balance.

2: Patient needs the continuous or intermittent support of one person to help with balance and coordination.

3: Patient requires verbal supervision or stand-by help from one person without physical contact.

4: Patient can walk independently on level ground, but requires help on stairs, slopes, or uneven surfaces.

5: Patient can walk independently anywhere.

Holden MK et al. [8]

\section{Comparisons between patients with and without independent walking}

Among the 128 patients, 65 could walk independently (FAC $\geq 4$; independent-walking group) at discharge and 63 could not (FAC <4; dependent-walking group). The two patient groups were significantly different in terms of age, duration from symptom onset to rehabilitation hospital admission, presence/absence of ventricular bleeding, hematoma volume, NIHSS scores, MMSE scores, and presence/absence of unilateral neglect (Table 2). Specifically, compared to the dependent-walking group, patients in the independent-walking group were younger and had shorter durations from symptom onset to rehabilitation hospital admission, less ventricular bleeding, smaller hematoma volumes, lower NIHSS scores, higher MMSE scores, and fewer instances of unilateral neglect.

\section{Decision-tree analysis}

Figure 2 depicts the decision tree. The positive classification rate in patients was $85.2 \%$, and the error rate by cross validation was 0.042 . The analysis revealed that the NIHSS score, MMSE score, and age were factors related to independent walking. In this model, the NIHSS score was placed in the first tier and patients were divided based on their score $(<6$ vs. $\geq 6)$. For patients with an NIHSS score $<6$, the second tier was divided based on the MMSE score $(<21$ vs. $\geq 21)$, while patients with an NIHSS score $\geq 6$ were divided based on age ( $<74$ vs. $\geq 74$ years). Regarding the third tier, patients with an MMSE score $<21$ were further divided according to the presence/absence of ventricular bleeding, while patients $<74$ years of age were divided according to the hematoma volume.

\section{Discussion}

The present study examined the relationships among evaluations performed upon admission to the rehabilitation hospital (i.e., after discharge from the acute-care hospital) and independent walking. Additionally, based on medical information frequently used in acute-care hospitals (CT images, NIHSS scores, MMSE scores), we performed a decisiontree analysis to investigate whether it is possible to predict independent walking upon admission to the rehabilitation hospital. Although a study indicated that patients' functional outcomes could be predicted based on their NIHSS and MMSE scores at the time of admission to an acutecare hospital [10], few studies have investigated walking outcomes using the evaluation methods performed at acute-care hospital discharge [3].

We found that the dependent- and independent-walking groups were significantly different in terms of age, duration from symptom onset to rehabilitation hospital admission, presence/absence of ventricular bleeding, hematoma volume, NIHSS score, MMSE score, and presence/absence of unilateral neglect. Fukiishi et al. [3] noted that among the factors related to walking ability in patients with thalamic hemorrhage, the patient's age, CT classification, hematoma volume, and state of consciousness at the time

Table 2 Comparisons between the independent- and dependent-walking groups

\begin{tabular}{llll}
\hline & Independent (FAC $\geq 4) n=65$ & Dependent (FAC $<4) n=63$ & $p$ value \\
\hline Age (years) & $65(48-84)$ & $71(40-93)$ & 0.0003 \\
Sex, male/female & $42 / 23$ & $33 / 30$ & 0.1595 \\
Duration from symptom onset to rehabilitation hospital admission (days) & $23(11-48)$ & $28(11-57)$ & 0.0045 \\
Lesion side, right/left & $30 / 35$ & $33 / 30$ & 0.4811 \\
Hematoma volume (mL) & $6(0.9-17.3)$ & $8.6(0.3-25.3)$ & $3 / 36 / 24$ \\
Hematoma type, I/I//II & $3 / 51 / 11$ & $48 / 15$ & 0.0038 \\
Ventricle bleeding, present/absent & $38 / 27$ & $10(2-23)$ & 0.234 \\
National Institutes of Health Stroke Scale & $5(1-17)$ & $20(6-30)$ & 0.0318 \\
Mini-Mental State Examination (/30) & $26(8-30)$ & $22 / 41$ & $<0.0001$ \\
Aphasia, present/absent & $20 / 45$ & $24 / 39$ & 0.0001 \\
Unilateral neglect, present/absent & $9 / 56$ & 0.0015 \\
\hline
\end{tabular}

Data are presented as the number or as the mean and range 


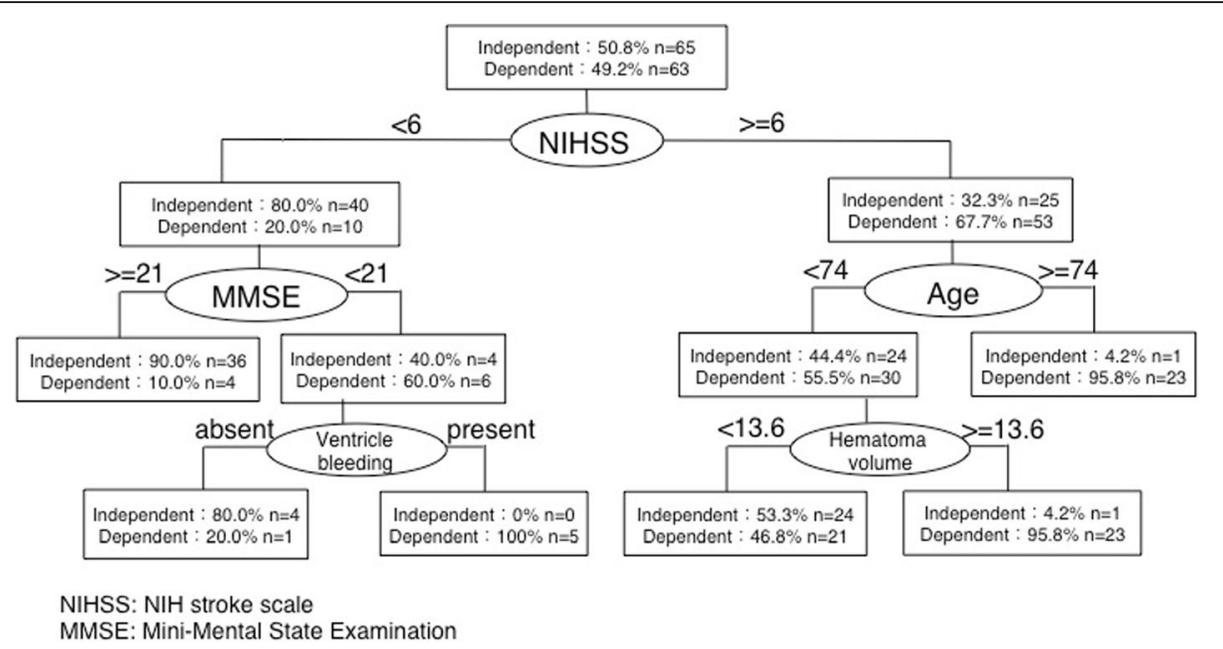

Fig. 2 Decision-tree analysis for factors related to walking independence in patients with thalamic hemorrhage. The National Institutes of Health Stroke Scale (NIHSS) score was placed in the first tier and patients were divided based on their score. For patients with an NIHSS score $<6$, the second tier was divided based on the Mini-Mental State Examination (MMSE) score, while patients with an NIHSS score $\geq 6$ were divided based on age. Regarding the third tier, patients with an MMSE score $<21$ were further divided according to the presence/absence of ventricular bleeding, while patients $<74$ years of age were divided according to the hematoma volume

of admission to an acute-care hospital are particularly important. Indeed, evidence shows that the greater the hematoma volume, the poorer the functional outcome [11]. Unilateral neglect is also known to inhibit independent walking, and patients with unilateral neglect have lower ADL scores than do patients without neglect [2]. Consistent with these previous studies, the present study also revealed that age, hematoma volume, and neurological severity, as measured with the NIHSS, were related to independent walking in patients with thalamic hemorrhage. Reports from rehabilitation hospitals show that there is a strong relationship between an improved functional outcome at rehabilitation hospital discharge and the early initiation of rehabilitation services $[12,13]$. Since no information on the rehabilitation interventions performed at the acute-care hospital was available in this study, this factor should be studied in the future.

Our decision-tree analysis revealed that the NIHSS and MMSE scores, hematoma volume, and presence/absence of ventricular bleeding were factors related to independent walking. By using the NIHSS and MMSE, which are standard, comprehensive evaluations performed at acute-care hospitals for patients with stroke, independent walking could be predicted in about $80 \%$ of the patients without using special evaluation scales like the Functional Independence Measure. Here, the MMSE was selected in the decision-tree analysis as a factor that was related to independent walking. This may be because patients with low MMSE scores need some assistance walking in the clinical setting, as they are unable to adapt to the environment and ultimately fall down. Such patients were given an FAC of 3 or less because they either required verbal supervision or stand-by help from one person. Therefore, patients with low MMSE scores often could not walk independently.

Our study suggests that independent walking can be estimated by adding the image-based diagnosis after stratification by NIHSS, MMSE, and age. Although the accuracy of predictions based only on neuroimaging data is not sufficiently high [14], the present study confirmed that neuroimaging data (hematoma volume and ventricular bleeding) are useful after stratification with neurologic symptoms using decision-tree analyses.

One limitation of the present study is that we utilized CT images. In order to improve the accuracy, future studies should consider the effects of microbleeds and asymptomatic cerebral infarctions using magnetic resonance images. Another limitation is that we did not consider the location of the lesion in the thalamus. This may be problematic, as different regions of the thalamus are involved in different functions. Specifically, the ventrolateral thalamus is involved in motor function, while the ventroposterolateral thalamus is involved in somatosensory function. Although we evaluated patients' neurological deficits using the NIHSS, as well as the hematoma type, additional studies that consider the lesion location should be performed in the future to confirm our findings.

\section{Conclusion}

Neuroimaging data, including the hematoma volume and presence of ventricular bleeding, along with age, neurological symptoms, and cognitive functions were useful for predicting the walking ability of patients with thalamic hemorrhage. 


\section{Abbreviations}

ADL: Activities of daily living; CT: Computed tomography; FAC: Functional ambulation category; MMSE: Mini-mental state examination; NIHSS: Nationa Institutes of Health Stroke Scale

\section{Acknowledgements}

We would like to thank all of the patients who participated in this study.

\section{Funding}

This work was supported by the "Research on Measures for Intractable Diseases" Project 2016: matching fund subsidy from the Ministry of Health, Labor, and Welfare.

\section{Availability of data and materials}

Data tendencies and dispersions supporting the conclusions of this study are provided within the manuscript. Raw data is available from the corresponding author upon reasonable request.

\section{Authors' contributions}

SH drafted the manuscript and SM analyzed the clinical data. HO, SO, and SS participated in the study design and helped to draft the manuscript. HH, ST, $\mathrm{RF}, \mathrm{KY}, \mathrm{IF}$, and NA participated in the medical examinations and made substantial contribution to acquisition of data. All authors read and approved the final manuscript.

\section{Ethics approval and consent to participate}

Approval was obtained from the Institutional Review Board at Fujita Health University (ID number: HM15-134). Written informed consent was obtained from all patients or their legally acceptable representatives following a thorough explanation of the study.

\section{Consent for publication}

Consent provided upon request.

\section{Competing interests}

The authors declare that they have no competing interests.

\section{Publisher's Note}

Springer Nature remains neutral with regard to jurisdictional claims in published maps and institutional affiliations.

Received: 30 April 2017 Accepted: 28 November 2017

Published online: 08 December 2017

\section{References}

1. Kobayashi S. Japan Standard Stroke Registry Study Group. Japanese Stroke Data Bank 2015. Tokyo: Nakayama Shoten Co., Ltd; 2015.

2. Maeshima S, Truman G, Smith DS, Dohi N, Itakura T, Komai N. Functional outcome following thalamic haemorrhage: relationship between motor and cognitive functions and ADL. Disabil Rehabil. 1997;19:459-64.

3. Fukiishi Y. Production of the outcome for a walking ability in thalamic hemorrhage patients from initial information: a trial by multivariate analysis. Jpn J Rehabil Med. 1987:24:169-74

4. Kanaya H, Saiki I, Ohuchi T, Kamata K, Endo H, Mizukami M, et al. Update on surgical treatment. In: Mizukami M, Kogure K, Kanaya H, editors. Hypertensive Intracerebral Hemorrhage. New York: Raven Press; 1983. p. 147-63.

5. Kothari RU, Brott T, Broderick JP, Marler JR, Barsan WG, Biller J, et al. The ABCs of measuring intracerebral hemorrhage volumes. Stroke. 1996;27:1304-5.

6. Brott T, Adams HP Jr, Olinger CP, Marler JR, Barsan WG, Biller J, et al. Measurements of acute cerebral infarction: a clinical examination scale. Stroke. 1989;20:864-70.

7. Folstein MF, Folstein SE, McHugh PR. "Mini-mental state". A practical method for grading the cognitive state of patients for the clinician. J Psychiatr Res. 1975;12:189-98.

8. Holden MK, Gill KM, Magliozzi MR, Nathan J, Piehl-Baker L. Clinical gait assessment in the neurologically impaired. Reliability and meaningfulness. Phys Ther. 1984;64:35-40

9. Brieman L, Friedmen J, Olshen R, Stone C. Classification and regression trees. Pacific Grove: Wadsworth; 1984.
10. Toshima M, Nishiya M, Hagiwara R. Factors affecting length of stay and discharge destination in patients with acute ischemic stroke. Jpn J Rehabil Med. 2001;38:268-76.

11. Toyoda O, Nakajima H, Kakegawa T, Nakajima S, Arai K, Kobayashi T. Relationship between hematoma volume and prognosis for thalamic hemorrhage. Neurol Med Chir. 1987;27:968-72.

12. Cifu DX, Stewart DG. Factors affecting functional outcome after stroke: a critical review of rehabilitation interventions. Arch Phys Med Rehabil. 1999; 80:S35-9.

13. Moon HI, Lee HJ, Yoon SY. Lesion location associated with balance recovery and gait velocity change after rehabilitation in stroke patients. Neuroradiology. 2017;59:609-18.

14. Matsuo H, Sonoda S, Maeshima S, Watanabe M, Sasaki S, Okuyama Y, et al. Contribution of physical impairment or imaging findings in the prediction of ADL outcome in stroke patients with middle cerebral artery infarction. Jpn J Compr Rehabil Sci. 2016;7:119-29.

\section{Submit your next manuscript to BioMed Central and we will help you at every step:}

- We accept pre-submission inquiries

- Our selector tool helps you to find the most relevant journal

- We provide round the clock customer support

- Convenient online submission

- Thorough peer review

- Inclusion in PubMed and all major indexing services

- Maximum visibility for your research

Submit your manuscript at www.biomedcentral.com/submit 